\title{
A CLASS OF EASILY PATH DELAY FAULT TESTABLE CIRCUITS
}

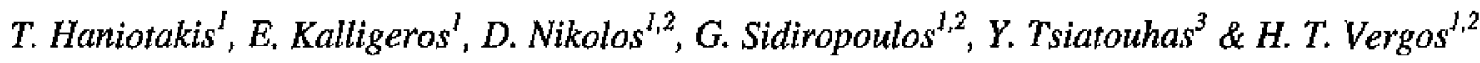 \\ ${ }^{1}$ Department of Computer Engineering and Informatics, University of Patras, 26 500, Greece \\ ${ }^{2}$ Computer Technology Institute, 3 Kolokotroni Str, 26221 Patras, Greece \\ ${ }^{3}$ ISD S.A, 22, K. Varnali Str, 15233 Maroussi, Athens, Greece
}

\begin{abstract}
The number of physical paths in a carty save or modified Booth multiplier, as well as in a non restoring cellular array divider is prohibitively large for testing all paths for delay faults. Besides, neither all paths are robustly testable nor a basis consisting of SPP-HFRT paths exists.

In this paper we present sufficient modifications of the above mentioned circuits so that a basis consisting of SPP-HFRT paths to exist. The cardinality of the derived basis is very small. Also, hardware and delay overhends due to the modifications are respectively small and negligible.
\end{abstract}

\section{INTRODUCTYON}

Imprecise delay modeling, the statistical variations of the parameters during the manufacturing process as well as the occurrence of physical defects in the integrated circuits result in chip malfunction at the desired speed. The path delay fault model [1] addresses distributed or accumulated delays due to the propagation through several lines, each affected by a delay defect. Two major problems are associated with path delay fault testing :

a) An excessively large number of physical paths needs to be tested. Usually it is not affordable to test all of them. For example the number of physical paths in a $32 \times 32$ Carry Save Array Multiplier is of the order of $10^{34}$ while in a non-restoring cellular array divider is of the onder of $10^{324}$.

b) Since the single fault assumption is not realistic for the path delay fault model (a single defect usually will affect a large number of paths), a robust test is usually required for detecting a path delay fault. However, for many circuits, a large number of path delay faults is not robustly testable,

A variety of path selection methods have been proposed [2-8] to alleviate problem a. above. The simplest delay-based approach is to select all paths whose calculated delay exceeds a specific threshold. However, the number of paths selected by this method is so large in general that all of the selected paths cannot be tested, especially in the case of optimized circuits [9]. The method given in [3] selects a set of paths such that for each interconnect $l$ of a given circuit the set contains at least one path with the largest calculated delay anong the paths through $l$. The number of the paths selected by this method is moderate. The method, recently proposed in [4], reduces the number of paths to be tested by judging which of two paths has the larger real delay. A common drawback of the above mentioned methods [3, 4] is that the selected paths may not be robustly testable (a large percentage of paths in a circuit is not robustly testable).

A number of functional approaches has also been proposed [5-8], In those, path selection is achieved by excluding paths that do not have to be tested functionally, such as unsensitizable paths, robustdependent paths etc. However, these approaches are not practical for large-sized circuits because computation time and the number of the selected paths ate quite large.

It has been shown in [2] that by mensuring the delays along a suitable very small set $\mathbf{R}$ of physical paths the propagation delays along any other path can be calculated (we will hereafter call such a set of paths a basis). However, to be able to measure the propagation delay along the $\mathbf{R}$ paths they must be Single Path Propagating - Hazard Free Robustly Testable (SPP. HFRT) [2]. Unfortunately for most circuits, a basis consisting of SPP-HFRT paths does not exist.

Almost all contemporary general and special purpose processors include a high speed multiplier and often a divider circuit. Testing them for path delay faults is a very difficult task due to: a) their excessively large number of physical paths and b) all path delay faults are not robustly testable. In this paper, we focus on $\mathbf{n} \times \mathbf{n}$ carry-save (CSM) and modified Booth (MBM) multipliers as well as the Non - Restoring Cellular Array Dividers (NRCADs), originally introduced in [10]. The methods given in $[3,4]$ can not be applied in the case of a CSM, MBM or an NRCAD, because many of the longer paths are neither robustly testable nor nonrobustly validatable. Also, a basis $R$, according to [2], consisting of SPP-HFRT paths does not exist for the above circuits.

In this paper modifications of the above circuits are proposed leading to a basis $\Delta$ consisting of SPP-HFRT paths. The delay overhead due to the modifications is negligible while the hardware overhead for practical size circuits is small. Under these modifications a basis of SPP-HFRT paths with small cardinality can be derived. However due to the prohibitively large number of paths 
of these circuits (for example the number of paths of the $32 \times 32 \mathrm{MBM}$ is equal to $2.4 \times 10^{15}$ ) it is impossible to calculate the delay along all paths in order to derive the maximum path delay. This problem can be overcome using the method proposed in [4] to determine a relatively small number of paths $\Pi$ the propagation delay along which must be calculated in order the maximum path delay of the circuit under test to be derived.

It has been shown in [11] that the fact that the circuit functions correctly at a speed does not imply that it will also function correctly at a lower speed. If we can test all primitive path delay faults of a circuit at a speed and during test application no delay fault is detected then the circuit functions correctly at any lower speed [11]. We will show in Section II that a circuit which does not have a strong delay - verification test set may have a basis consisting of SPP-HFRT paths. In this case measuring the delays along the paths of the basis and calculating the propagation delay along all paths included in primitive faults we derive the maximum delay of the circuit (as well as the maximum speed). Then the circuit functions correctly for all lower speeds.

We consider the inputs and outputs of the maltiplier / divider as primary inputs (PIs) and primary outputs (POs) of the chip. In the case that the circuit is embedded in a larger module, its inputs and outputs can easily be made accessible by the PIs and the POs of the module using, for example, the method proposed in [12].

\section{SPP-HFR'T PATHS AND DELAY-VERIFIABLE CIRCUITS}

A two pattern test $T=\left\langle V_{1}, V_{2}\right\rangle$ is said to be a robust delay test for a path $\mathrm{P}$, for a rising or falling transition at the input of the path, if and only if, when $P$ is faulty and test $T$ is applied, the circuit output is different from the expected state at sampling time, independent of the delays along gate inputs not on $P$ [13]. A robust test that propagates the fault effect through only a single path to an output in the circuit will be called a Single-Path Propagating Robust Test (SPP-RT) for that output. A robust test is said to be a Hazard-Free Robust Test (HFRT) if no hazards can occur on the tested path during the application of the test, regardless of the gate delay values. Robust tests may not exist for all path delay faults in an arbittary circuit.

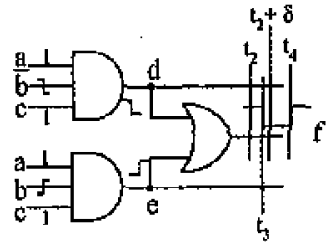

Figure 1.a

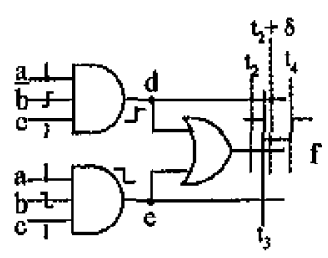

Figure 1.b
It has been shown in [11] that the fact that a circuit functions correctly at a speed does not imply that it will also function contectly at a lower speed. A set of path delay tests is called a strong delay-verification test set if the correct response of the CUT at a speed implies correct operation at any lower speed [11], A circuit which has a strong delay-verification test set is called a delay-verifiable circuit [11]. Figure 1 [11] shows a circuit which does not have a strong delay-verification test set. All faults except $\uparrow \overline{\text { bdf }}, \downarrow \bar{b} d f, \uparrow$ bef and $\downarrow$ bef are testable by robust tests, In this case, even the exhaustive set consisting of all the vector paits is not a strong delay verification test set. The signal values in the circuit for the two tests $\langle 101,111\rangle$ and $\langle 111,101\rangle$ are shown in Figures 1.a and $1 . b$, respectively. If there are no path delay faults, any output pulse that may occur will occur before the sampling time $t_{2}$. Faults on the paths from $b$ and $\bar{b}$ may result in an output pulse occurring later. Such faults may or may not be detected at time $t_{2}$. Therefore, the correct response for these two tests only guarantees that the circuit will operate cortectly if its period is set to the test period $\tau$, but the delayed pulse due to the path delay fault may cause incorrect operation at a lower speed.

However, although a strong-delay verification test set does not exist for the circuit of Figure 1 under the definition given in [11], we will show below that we can calculate the maximum speed of the circuit and that for any lower speed the circuit will function correctly. The propagation delay along the paths ad, adf, ae, aef, $\bar{b} d$ and be which are SPP-HFRT can be measured applying the test vector pairs $<001,101\rangle,<001,101\rangle,<011$, $111\rangle,\langle 011, \quad 111\rangle,\langle 101,111\rangle$ and $\langle 101,11\rangle$ respectively. Then the propagation delay, pd, along the paths $\bar{b}$ df and bef can be calculated by : $\operatorname{pd}(\uparrow \overline{b d f})=p d(\uparrow \overline{b d})+p d(\uparrow$ adf $)-p d(\uparrow$ ad $)$, $\operatorname{pd}(\downarrow \overline{\text { bdf }})=\operatorname{pd}(\downarrow \overline{\mathrm{bd}})+\operatorname{pd}(\downarrow$ adf $)-\operatorname{pd}(\downarrow$ ad $)$, $\operatorname{pd}(\uparrow$ bef $)=\operatorname{pd}(\uparrow$ be $)+\operatorname{pd}(\uparrow$ aef $)-\operatorname{pd}(\uparrow$ ae $)$ and $\operatorname{pd}(\downarrow$ bef $)=\operatorname{pd}(\downarrow$ be $)+\operatorname{pd}(\downarrow$ aef $)-\operatorname{pd}(\downarrow$ ae $)$.

For the output waveforms of figures 1.a and 1.b we get $\quad \operatorname{pd}(\uparrow \overline{b d f})=t_{4}, \quad \operatorname{pd}(\downarrow \bar{b} d f)=t_{3}, \quad \operatorname{pd}(\uparrow$ bef $)=t_{4}$, pd $(\downarrow$ bef $)=t_{3}$, therefore the maximum delay of the circuit is equal to $t_{4}$ and for lower speeds the circuit will function correctly. From the above discussion it becomes evident that for a circuit having a basis consisting only of SPP-HFRT paths we can calculate the delay along all paths or along the paths included in primitive faults [11] and the calculated maximum propagation delay implies that the circuit will function correctly for any lower speed. 


\section{III, CARRY - SAVE MULTIPLIERS}

An $n \times m$ CSM is a circuit with inputs $\left(A_{1}, A_{2}, \ldots A_{n}\right)$ and $\left(B_{1}, B_{2}, \ldots, B_{m}\right)$ and outputs $\left(O_{1}, O_{2}, \ldots, O_{n+m}\right)$. Figure 2 presents the $4 \times 4$ carry save multiplier. We consider that the multiplier consists of two blocks. The first block $\mathrm{D}_{0}$ consists of the network of carry save adders and the associated logic. The second block $D_{1}$ is an (n-1)-bit adder, which can be implemented as a ripple carry or group carry look ahead adder.

In [12] we have shown that using multiplexers for making the inputs and outputs of the embedded blocks accessible by the primary ports of the circuit, the path delay fault testing of the circuit is reduced to the path delay fault testing of the blocks that constitute it. By adding multiplexers in the original CSM design (Figure 3 ), we can manipulate the two blocks, $D_{0}$ and $D_{1}$, individually. We will hereafter deal only with the path delay fault testing of $D_{0}$, since efficient path delay fault testing techniques of both ripple-carty and carry look ahead implementations of $D_{1}$ have been presented in [14]. A basis consisting only of SPP-HFRT paths of $D_{0}$ does not exist. Hence some additional modifications of $D_{0}$ are required. Specifically, the half adders of $D_{0}$, that is, the adders of the first row, are substituted by full adders. The extra input of the leftmost adder, of the first row, is driven by a test input $t_{1}$. The extra inputs of the rest adders of the first row are driven alternately by the $t_{2}$ and $t_{3}$ test inputs. During nomal circuit operation all three test inputs $t_{1}, t_{2}$ and $t_{3}$ are driven to 0 .

The selection of the SPP-HFRT paths of a basis for the CSM after the above modifications was presented in [15].

\section{MODIFIED BOOTH MULTIPLIERS}

We consider $\mathrm{n} x \mathrm{n}$ modified Booth multipliers where $\mathrm{n}=2^{\mathrm{k}}$, with sign generate. An $\mathrm{n} \times \mathrm{n} M B M$ is $\mathrm{a}$ combinational circuit with inputs $\left(A_{1}, A_{2}, \ldots, A_{n}\right)$ and $\left(B_{1}\right.$, $\left.B_{2}, \ldots, B_{n}\right)$ and outputs $\left(P_{1}, P_{2}, \ldots, P_{2 n}\right)$. Figure 4 presents the $8 \times 8 \mathrm{MBM}$.

We also consider that the multiplier consists of two blocks. The first block $D_{0}$ contains the logic that forms and adds the partial products and consists of two parts :

a) The first part is responsible for the 2-bit recoding function, and is implemented by the $\mathrm{n} / 2$ cells at the left end, named I-cells. The implementation of an Icell is presented in Figure 5 .

b) The second part is responsible for the generation and addition of the partial sums and is implemented by:

b1) $(\mathrm{n}-1)^{*}(\mathrm{n} / 2)$ cells, named ps-cells. The implementation of a ps-cell is presented in Figure 6.

b2) $n / 2$ cells, named 1_ps-cells, An 1_ps-cell is the leftmost cell in a ps-cell row. It can be a normal pscell with an inverter at the output and its $I_{4}$ and $I_{5}$ inputs driven from the same signal $\left(A_{11}\right)$. Since such
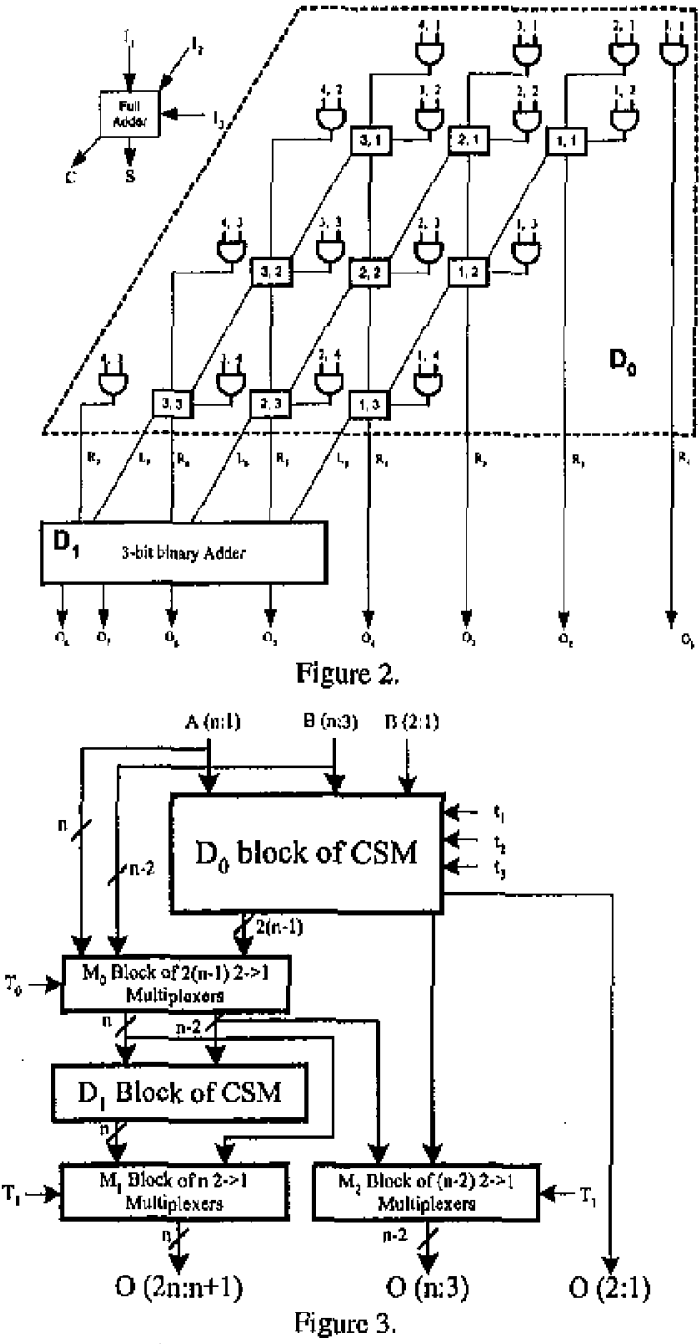

modifications result in an non robustly testable 1_pscell, in Figure 7 we present the implementation of a robustly testable 1_ps-cell.

b3) $n / 2$ cells, named r_ps-cells. An r_ps-cell is the rightmost cell in a ps-cell row. It can be designed as a normal ps-cell with its $I_{s}$ input connected to ground. This results in a non-robustly testable cell. A robustly testable implementation for an $\mathbf{r}$ _ps-cell is shown in Figure 8.

b4) $\left.(\mathrm{n}-1)^{*}[(\mathrm{n} / 2)-2)\right]+1$ full adders, We consider every full adder implemented as in [14].

b5) $n+(n / 2)-3$ half adders.

The second block $D_{1}$ is an $\left(2^{*} n\right)$-bit adder which forms the final result. $D_{1}$ can be implemented as a ripple carry or group carry look ahead adder.

Using multiplexers for making the inputs and outputs of the embedded blocks controllable and observable 


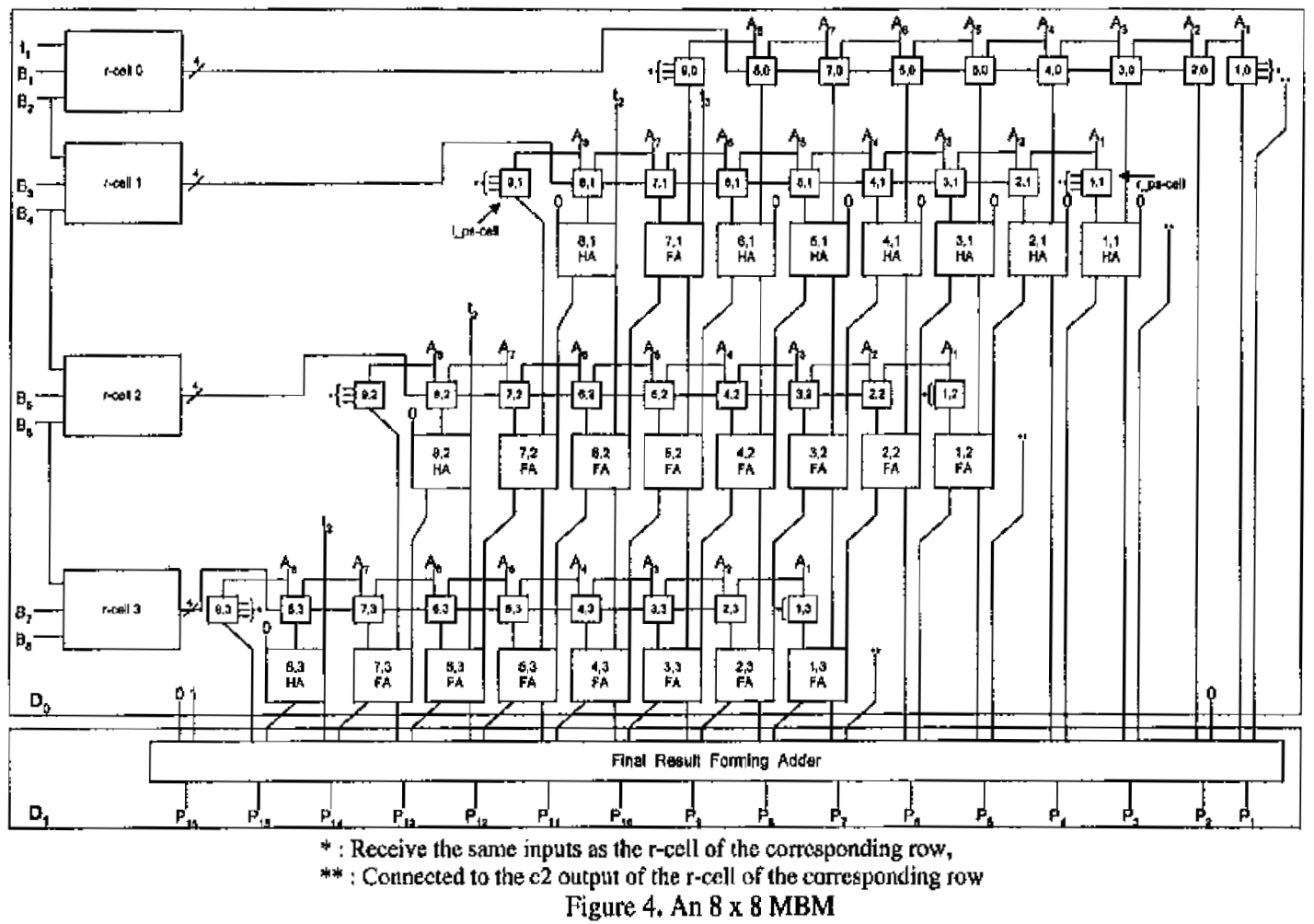

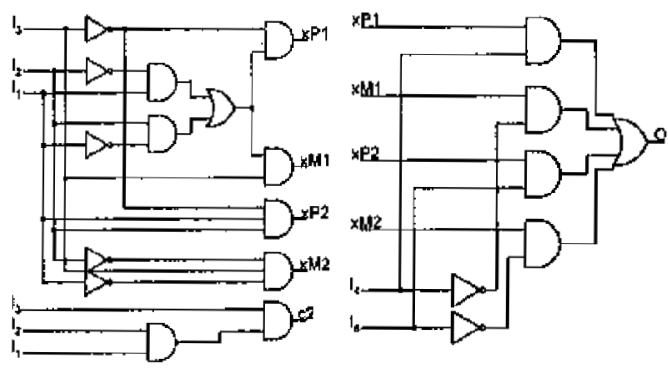

Figure 5.
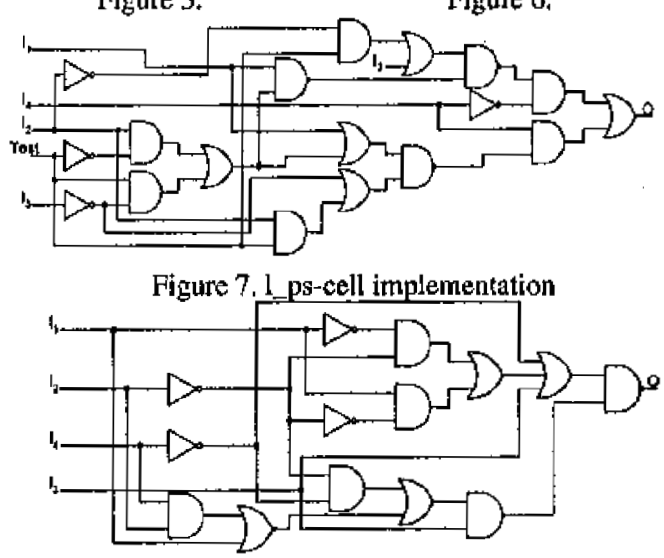

Figure 8. r_ps-cell implementation

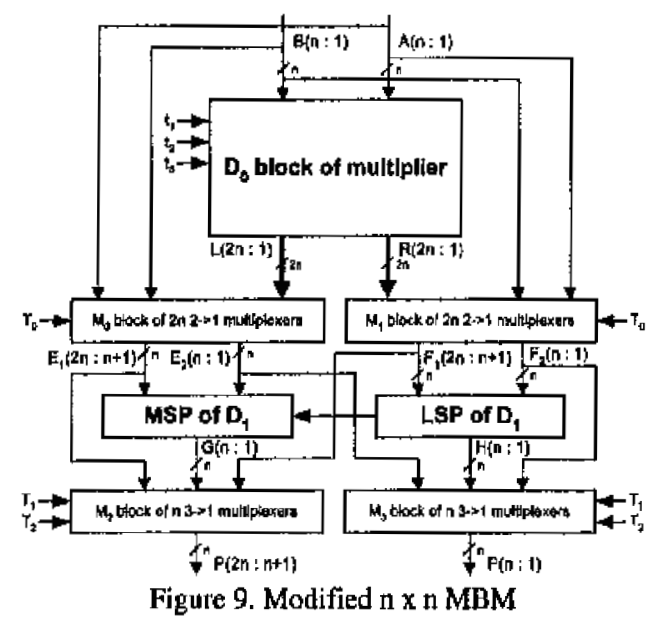

respectively by the primary ports of the circuit, the path delay fault testing of the circuit is reduced to the path delay fault testing of the blocks that constitute it [12]. In Figure 9, we show how this technique can be applied to n $x \cap$ MBMs. By adding multiplexers in the original MBM design, we can manipulate the blocks, $D_{0}$ and $D_{b}$, individually [12].

Due to the fact that both the least (LSP) and the most (MSP) parts of $D_{1}$ under the above modifications receive the same test inputs, some of the paths that in [14] are tested in parallel, in this case must be tested explicitly. In the case that each part of $D_{1}$ is an ILA with four or more 


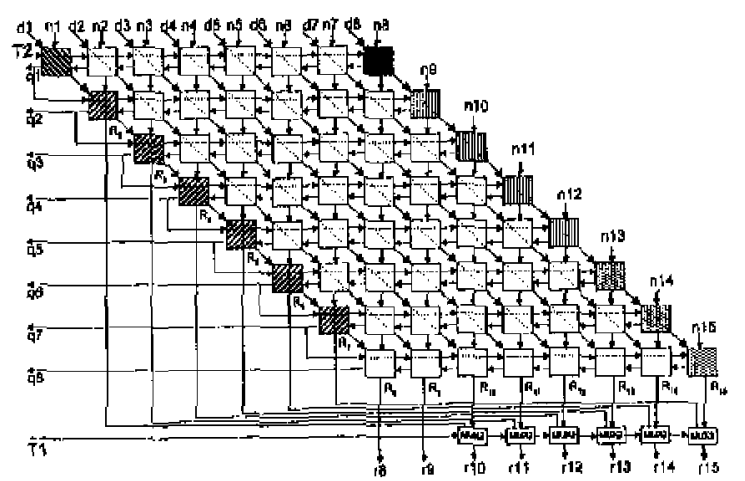

Figure $10.8 \times 8 \mathrm{NRCAD}$.

cells, the paths that should be tested explicitly are only those including carry propagation from LSP to MSP, while for all the rest parallel robust path delay fault testing can be carried as in [14].

The selection of the SPP-HFRT paths of a basis for the CSM after the above modifications was given in [16].

\section{V; NON-RESTORING CELLULAR ARRAY DIVIDERS}

An $N \times N$ NRCAD is a combinational circuit with inputs the nominator $\left(n_{1}, n_{2}, \ldots, n_{2 N-1}\right)$ and the denominator $\left(\mathrm{d}_{1}, \mathrm{~d}_{2}, \ldots \mathrm{d}_{\mathrm{N}}\right)$ and outputs the quotient $\left(\mathrm{q}_{\mathrm{l}}\right.$. $\left.q_{2}, \ldots q_{N}\right)$ and the remainder $\left(r_{N}, r_{N+2}, \ldots, r_{2 N-1}\right)$. Figure 10 presents the $8 \times 8$ NRCAD (ignore the multiplexers at the bottom of Figure 10). The dashed lines indicate propagation of the signals to the next cell in either horizontal or diagonal direction. The NRCAD is formed as a two dimensional matrix of identical logic cells. The implementation of each cell of the divider considered in this paper is presented in Figure $11 . \mathrm{a}$ and requires 19 gates, without considering the gate with the bold out]ine. Since the first row cells have their $P$ input driven by logical 1, they can be implemented by 15 gates. Since the leftmost cell of each row, except the last, does not produce an $S$ output it can be implemented by 9 gates as shown in figure 11.b. The upper and leftmost cell can be implemented by 5 gates. Since the rightmost cell of each row, except the first, has connected $P$ and $C_{i}$ inputs, it can be implemented by 9 gates as shown in Figure 11.c, without considering the gate with the bold outline. The upper and rightmost cell requires only 8 gates. Summarizing the above, we can express the total area of the divider in gates as $A_{\text {total }}=19 *[(\mathrm{~N}-1) *(\mathrm{~N}$ $2)+1]+15^{*}(\mathrm{~N}-2)+9 *(\mathrm{~N}-2)+5+9 *(\mathrm{~N}-1)+8$.

In the sequel we propose several design modifications for making the NRCAD design easily testable. Excluding the leftmost cells of all rows and the cells of last row, we augment every other cell with an extra AND gate (the AND gate with the bold outline of figures 11.a, 11.c). An extra test input $T_{0}$ is used to drive the second input of the
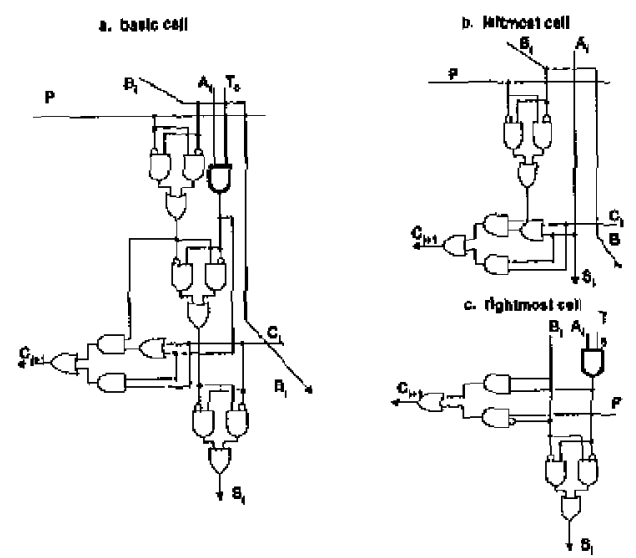

Figure 11. Building blocks of the NRCAD

added AND gate for all cells. $\mathrm{T}_{0}$ is only used during testing. During normal citcuit operation $T_{0}$ is driven to 1 . The hardware overhead of the addition of the AND gate in terms of gate equivalents is $(\mathrm{N}-1)^{2} / \mathrm{A}_{\text {1oral. }}$ The above relation, for $\mathrm{N}=8,16$ and 32 leads to a hardware overhead of $4.7,5.0$ and $5.14 \%$ respectively. The critical path of the design is from a primary input of the upper and rightmost CAS cell, through the carry chains of each of the $\mathbf{N}$ levels of the NRCAD. The chains of two adjacent levels are connected together through the $C_{i+1}$ output of the leftmost cell which is connected to $\mathrm{P}$ input of the rightmost cell of next row. The AND gates that we have added do not add any delay on this critical path and all the rest paths that include any sub-path along an added AND gate have smaller propagation delay times than this critical path.For providing observability of the $S$ output of the next to the leftmost cell of each row, excluding the first and last rows, we include $\mathrm{N}-2$ 2 $2>1$ multiplexers to the NRCAD design. All these multiplexers are controlled by the same test input $T_{1}$ and connected as shown in Figure 10. $\mathrm{T}_{\mathrm{l}}$ during normal operation is driven to 1 and the remainder bits are observable at the primary outputs, whereas during testing of specific paths is driven to 0 and the $S$ outputs of the next to the leftmost cells become observable at the remainter outputs of the divider. The outpul of the multiplexer which drives the $\mathrm{r}_{\mathrm{i}+\mathrm{N}}$ primary output is denoted as $O_{i+N}$. Since the hardware implementation of the multiplexer requires 4 gate equivalents, the hardware overhead due to multiplexer insertion is: $4^{*}(\mathrm{~N}-2) / \mathrm{A}_{\text {total }}$. This relation, for $\mathrm{N}=8,16$ and 32 leads to a hardware overthead of $2.3,1.4$ and $0,64 \%$ respectively, Our simulations showed that the insertion of the multiplexers does not cause any delay overhead.

For providing controllability of the $P$ input for the cells of the first row, we drive all these inputs by a third test input $T_{2} . T_{2}$ is set to 1 for normal circuit operation and is occasionally driven to 0 during testing. The hardware overhead of this change is equal to 4 gates per 
cell of the first row, Thus the hardware overhead is $\left(4^{*} \mathrm{~N}\right) / \mathrm{A}_{\text {total }}$ or equivalently $3.0,1.4$ and $0.68 \%$ respectively for $\mathrm{N}=8,16$ and 32 . This change may increase the critical path of the design by a time equal to the difference of the worst propagation delays between an XOR gate and an inverter, which is overall a negligible delay.

After the above modififctaions the selection of an SPP-HFRT basis can be done as presented in [17],

\section{CONCLUSIONS}

The hardware overhead for CSM, MBM and NRCAD of various sizes are presented in Tables I, II and III respectively. We can see that for multipliers and dividers of practical sizes the hardware overhead is very small. The delay overhead of the easily path delay fault testable multipliers is equal to two gate levels, while the delay overhead for the dividers is negligible. From Tables I, II and III we can easily see that the number of paths along which we have to measure the propagation delay is small. However, the number of paths along which the propagation delay must be calculated is prohibitively Jarge. This problem can be overcome by calculating only the delays along the paths included in primitive faults. In this way we derive the maximum speed of the circuit and as we have shown we also ensure that the citcuit will function correctly at all lower speeds.

To the best of our knowledge robustly path - delay fault testable or delay verifiable CSM, MBM as well as NRCAD designs have not been reported in the open literature. Therefore the proposed circuits are the only known easily path delay fault testable multiplies and divider circuits.

\section{References}

[1] G. L. Smith, "Model for Delay Faults Based upon Paths", Proc, ITC - 85, pp. 342 - 349 .

[2] J. D. Lesser and J. J. Shedletsky. "An Experimental Delay Test Generator for LSI Logic", IEEE Trans. on Computers, C-29 (3), March 1980, pp. $235-248$.

[3] W.-N. Li, S. M. Reddy, and S. K. Sahni. "On path selection on combinational logic citcuits", IEEE Trans. on CAD, pp. 56-63. Jan. 1989.

[4] S. Tani, et,al, "Efficient Path Selection for Delay Testing Based on Partial Path Evaluation", Proc of $16^{\text {th }}$ IEEE VLSI Test Symp. pp. 188 - 193, 1998.

[5] K.-T, Cheng and H.-C. Chen. "Delays testing for non mobust untestable circuits", Proc. of Int. Test Conf., pp. 954-961. IEEE, 1993.

[6] W, K, Lan, et. al., "Delay fault coverage and performance tradeoffs", Proc. of the $30^{\text {th }}$ Design Automation Conf. , pp. 446-452. ACM//EEE, 1993.

[7] M. A. Gharaybeh, M. L. Bushnell, and V. D. Agrawal. "Classification and test generation for path-delay faults using single stuck-fault tests", Proc. of Int. Test Conf., pp.
139-148. IEEE, 1995.

[8] U. Sparmann, et. al.,"Fast identification of robust dependent path delay faults', Proc. of the $32^{\text {th }}$ Design Automation Conf., pp. 119-125. ACM-IEEE, 1995.

[9] T. W. Williams, et. al., "The interdependence between delay optimization of synthesized networks and testing", Proc. of the $28^{\text {th }}$ DAC, pp. 87-92. ACM/IEEE, 1991.

[10] H. H. Guild, "Some Cellular Logic Arrays for Nonrestoring Binary Division", The Radio and Elec. Engr., vol. 39, June 1970, pp. 345 - 348 .

[11] W. Ke and P. R. Menon, "Synthesis of Delay - Verifiable Combinational Circuits", IEEE Trans. on Computers, Feb. 1995, pp. 213-222.

[12] D. Nikolos et al., "Path Delay Fault Testing of ICs with Embedded Intellectual Property Blocks", Proc. of DATE '99, March 1999, pp. $112-116$.

[13] C. J. Lin and M, Reddy, "On Delay Fault Testing in Logic Circuits", IEEE Trans. on CAD, Sep. 1987, pp. 694-703.

[14] T. Haniotakis, Y. Tsiatouhas and D. Nikolos, "C-Testable One-Dimensional ILAs with Respect to Path Delay Faults : Theory and Applications", Proc, of IEEE Int. Symp. on Defect and Fault Tolerance in VLSI Systems, November 1998, pp. $155-163$.

[15] Th. Haniotakis, et al," "Easily Testable Carry Save Multipliers with respect to Path Delay Faults", $2^{\text {nd }}$ Electronic Circuits and Systems Conference, September 6-8 1999, Bratislava, Slovakia, pp. 13-16.

[16] E. Kalligeros, ef al, "Path Delay Fault Testable Modified Booth Multipliers", Proc. of the XIV Design of Circuits and Integrated Systems Conference (DClS '99), Nov. 1619 1999, Palma de Mallorea, Spain, pp. 301 - 306.

[17] G. Sidiropoulos, et al,, "Easily Path Delay Fault Testable Non-Restoring Cellular Array Dividers", Proc. of ATS 99, Nov. 16-18 1999, Shanghai, China, pp. 47 - 52.

Table I. CSM Comparisons

\begin{tabular}{|c|c|c|c|c|}
\hline $\begin{array}{c}\text { CSM } \\
\text { size }\end{array}$ & $\begin{array}{c}\text { Hardware } \\
\text { Overliead (\%) }\end{array}$ & $\begin{array}{c}\text { Number of } \\
\text { plysical paths }\end{array}$ & $\begin{array}{c}\text { Number of paths } \\
\text { to be tested }\end{array}$ & $\begin{array}{c}\text { Reduction } \\
\%\end{array}$ \\
\hline $8 \times 8$ & 17.93 & $5.825 \times 10^{8}$ & 1032 & 99.9998 \\
\hline $16 \times 16$ & 8.64 & $3.189 \times 10^{17}$ & 4392 & $\approx 100$ \\
\hline $32 \times 32$ & 4,24 & $6,245 \times 10^{34}$ & 18408 & $\approx 100$ \\
\hline $64 \times 64$ & 2,10 & $2,142 \times 10^{69}$ & 75624 & $\approx 100$ \\
\hline
\end{tabular}

Table II. MBM Comparisons

\begin{tabular}{|c|c|c|c|c|}
\hline $\begin{array}{c}\text { CSM } \\
\text { size }\end{array}$ & $\begin{array}{c}\text { Hardware } \\
\text { Overhead (\%) }\end{array}$ & $\begin{array}{c}\text { Number of } \\
\text { physical paths }\end{array}$ & $\begin{array}{c}\text { Number of paths Reduction } \\
\text { to be tested }\end{array}$ & $\%$ \\
\hline $16 \times 16$ & 12.4 & $1.5^{*} 10^{\%}$ & 7222 & $\approx 100$ \\
\hline $32 \times 32$ & 6.5 & $2.4^{*} 10^{15}$ & 18654 & $\approx 100$ \\
\hline $64 \times 64$ & 3.3 & $2.3^{*} 10^{27}$ & 65326 & $\approx 100$ \\
\hline
\end{tabular}

Table III. NRCAD Comparisons

\begin{tabular}{|c|c|c|c|c|}
\hline $\begin{array}{c}\text { CSM } \\
\text { size }\end{array}$ & $\begin{array}{c}\text { Hardware } \\
\text { Overhead (\%) }\end{array}$ & $\begin{array}{c}\text { Number of } \\
\text { phycal paths }\end{array}$ & $\begin{array}{c}\text { Number of path } \\
\text { to be tested }\end{array}$ & $\begin{array}{c}\text { \%otion } \\
\%\end{array}$ \\
\hline $8 \times 8$ & 10 & $3,7 \times 10^{21}$ & 735 & $\approx 100$ \\
\hline $16 \times 16$ & 7.8 & $5,3 \times 10^{80}$ & 3231 & $\approx 100$ \\
\hline $32 \times 32$ & 6.5 & $6,4 \times 10^{324}$ & 13599 & $\approx 100$ \\
\hline
\end{tabular}

